\title{
On Some Physical Properties of the Golgi Complex in the Living Cell as Revealed by Micro-operations under the Phase Microscope
}

\author{
By \\ Shunzo Takagi, Hideo Masuda and Masayuki Tagawa \\ Department of Biology, University of Osaka Prefecture, Sakai, Osaka \\ (Director: Prof. ShunzoTakagi)
}

That the Golgi complex viewed under the phase microscope was composed of some structural elements was first noted in isolated epididymal cells by Da 1 to $\mathrm{n}$ and Felix $(1952,1953)$ and in plasma cells by $\mathrm{H}$ a naoka (1954). A Golgi area with a characteristic appearance is usually more clearly discernible in well-spread cultured cells and is found to contain some formed components, which appear darker or brighter than the matrix according to the optics used and are described in various ways by the authors $(\mathrm{C} h$ è $\mathrm{v} \mathrm{re}$ m on t 1956; Frederic 1956 ; R ose and P o merat 1960 ; R o se 1961, 1962). In our previous study with cultured fibroblasts, myoblasts and the other cells, they were described as filaments or threads. Besides being intensely osmiophilic, the Golgi threads resemble mitochondria in vital staining with janus green and in staining after fixation with Altmann's acid fuchsin. But, both in living cells under the phase microscope and in stained preparations, they are usually more slender than the mitochondria of the cells and also distinguishable from the latter in being blurred in contour (T a k a g i and M a suda 1956 ; Tak a gi 1958 ; T a ka gi, K it a d a, $\mathrm{M} \mathrm{a} \mathrm{suda}$ and $\mathrm{Tagaw}$ a 1961). It seemed reasonable to suppose that in view of their microscopic appearance and dimensions they represent the double membranes, which are maintained to be the main constituents of the Golgi complex by the electron microscopists.

An extensive micrurgical study on cultured cells was made by $\mathrm{Chambers}$ and Fell (1931), but the Golgi elements could not be seen and their changes accompanying cell injuries could not be followed at that time with the use of dark-field illumination. In the present report, using cells cultured from the heart and the liver of chick embryos as the materials, some series of micro-operations 
were made under the phase microscope to learn some of the physical properties of the Golgi threads and the zone in which they are embedded, i. e., the Golgi area, the results of which will be described in the following lines.

\section{Materials and Methods}

Well-spread cells, the Golgi area of which was clearly indicated, were selected from among fibroblastic cells and hepatic epithelial cells cultured respectively from the chick heart and liver. The culture medium consisted of bovine serum $20 \mathrm{cc}$, Hanks balanced salt solution $80 \mathrm{cc}$, lactalbumin hydrolysate $0.25 \mathrm{~g}$ and yeast extract $0.05 \mathrm{~g}$. It was adjusted at a $\mathrm{pH}$ between $7.2-7.6$ with phenol red as the indicator. Cells were dissociated in 0.05 per cent trypsin solution and cultivated over 7 days on coverslips according to the method of monolayer culture. The selected coverslip was mounted on two glass supports, which were $2.8 \mathrm{~mm}$ thick and cemented to a microscope slide with Canada balsam, and the open-ended chamber thus formed was filled with Hanks solution adjusted at a $\mathrm{pH}$ between 7.2-7.6.

The preparation was placed on the stage of a phase contrast microscope equipped with a long working distance condenser and a $40 \times$ NDM objective (Tiyoda Optical Co.). The microneedle was driven with a micromanipulator of de Fonbrune type (Takashima Co.). The whole apparatus was placed in a thermo-regulated warm box at $36^{\circ} \pm 0.5^{\circ} \mathrm{C}$. Photographic records were taken of the cell before and after it was operated on.

\section{Observations and Experiments}

Our observations and experiments were made mainly on the Golgi complex and the Golgi area, i. e., the zone where it is included, but studies were made also on the general cytoplasm for purposes of comparison. The microscopic details of the Golgi area are more clearly indicated in dying or dead cells, which have suffered a severe mechanical injury, than in healthy living cells. Observations on these cells, therefore, will be described following those of the healthy cells. Next will be reported the experiments involving micro-operations on the Golgi complex, the Golgi area and the remaining parts of the cytoplasm in the living cells. All the statements without special notice are exclusively on both the fibroblastic 
cells and the hepatic epithelial cells which were employed as the materials.

(1) The Golgi complex in the healthy living cells.

The area where the Golgi elements are contained is a more or less clearly circumscribed region of the cytoplasm and is usually situated on the side of the nucleus which is provided with a broader zone of the cytoplasm. In many cells, it appears darker than the adjacent regions of the cytoplasm. It is easily recognized, as is of ten the case, when it is surrounded by numerous lipid droplets except on the side of the nucleus (Figs. 1, 3, 7 and 13).

The characteristic and universal constituents of the Golgi area which are visible under the phase microscope are filamentous or membranous structures with a fluffy contour. When followed by carefully adjusting the focus of the microscope, they are seen in some cells to run in all directions, but in many other cells with an ovoid nucleus most of them run parallel to the longer axis of the nucleus. That they represent the Golgi double membranes or lamellae or stacks of the lamellae will be evidenced by the features they present in dying and dead cells to be described in the next section. The Golgi lamellae are more slender and usually appear less phase-dark than the mitochondria and are fluffy in outline. Besides, they are somewhat different in staining properties from the mitochondria as was indicated in our previous papers.

Other inclusions most frequently met with in the Golgi area are lipid droplets. These are either a number of small droplets which appear like dark grains or a few medium-sized or large spherules which appear bright. Their lipid nature is evident as they are stained with sudan black ( $\mathrm{T}$ a k a $\mathrm{g}$, K it a d a, M a s u d and T ag a w a 1961). Fluid vacuoles, small or often medium-sized, are localized in the Golgi area of some cells. Although a few mitochondria could sometimes be found in this area ( $\mathrm{Takagi}$ and M a s u d a 1956), it was not the case in any of our present materials.

(2) The Golgi complex in the dying and dead cells.

When a cell is severely injured by a microneedle, it shows a series of changes eventually leading to death. For example, if a cut is made violently through a part of the cytoplasm, whereby the neighboring regions are inevitably stretched, or the nucleus is punctured, the cell will die unexceptionally. Formation of lobopodia or rounding up by retraction of cell processes, which is characteristic of the living cell as it responds to a stimulus, is of course not observable any more in dead cells (Compare Figs. 7 and 8, Figs. 
13 and 14).

Most remarkable in dying and dead cells is the fact that the nuclear membrane and the nucleolus become far more accentuated in visibility than in uninjured ones. In the cytoplasm, the Golgi lamellae, mitochondria and lipid droplets also greatly increase in contrast (Compare Figs. 3 and 4). In some cells, elements of the endoplasmic reticulum come to be visualized. Such alterations seem to be due at least in part to a decrease in hydration of the membranous structures of the cell.

Phase-lucent vacuoles of small or medium sizes in the Golgi area of the living cells often become lost sight of when these are injured and die. This is also the case with similarly looking vacuoles in the general cytoplasm. However, it sometimes occurs also in severely injured, dying cells that rather large vacuoles are newly formed in the general cytoplasm, more frequently close to the nucleus.

The Golgi lamellae which are usually only vaguely visible in the Golgi area rapidly increase in contrast in the cells dying or dead after a severe injury, and appear like a cluster of phase-dark threads. For an example, in the cell illustrated in Figs. 3 and 4, of which the nucleus has been punctured, the Golgi lamellae are very manifest after the operation, although they are slightly removed from their original position following a slight modification in form of the cell. That the phase-dark threads in the dying cell representing the Golgi lamellae do appear exactly in the zone of cytoplasm which is indicated as the Golgi area in the living condition is well exemplified in the cell shown in Figs. 5 and 6, where the Golgi area had two processes embracing a clump of lipid droplets. In each of the two cells (Figs. 4 and 6), the whole assembly of the Golgi lamellae or threads resembles at the first glance the Golgi apparatus familiar to us in metallic impregnation preparations.

The Golgi lamellae in the dead cells are, as they are in the living cells, more slender and less phase-dark than the mitochondria and rugged in outline. But, as they are far more distinct than in the living cells, they can now be studied fairly precisely in their arrangement and detailed morphology. The Golgi lamellae are observed to be like threads in optical section and the threads run in various directions. But, in many cells with an elongate nucleus, most of them run parallel to the nuclear membrane and the rest are orientated in different directions. It is evident that some Golgi 
threads divide themselves into branches. These come into contact, or anastomose, with each other. The Golgi threads thus take on the appearance of a net in certain instances.

In a few of the cells killed as a result of micro-operation, the Golgi lamellae were seen slightly swollen, while in some other cells, the mitochondria were swollen but the Golgi lamellae were not. In general, the cytoplasmic organelles and the other inclusions did not show any great tendency to swell in dying or dead cells at least during the course of observation.

(3) The Golgi complex manipulated in the living cell and the physical state of the Golgi complex.

All the experiments stated in the following paragraphs were made on living cells which were provided with manifest reactivity to stimulation.

A small tear was made through a part of the Golgi area. It was soon recovered by flowing together of the matrix and seemingly by formation of a new surface film. Even when a small phaselucent hole was made by the microneedle through the whole thickness of the Golgi area of a flattened cell, it was soon closed. When a cut of a considerable length was made in the area, the wound remained as a broad phase-lucent streak with a dark band on either side, which was formed by local thickening of the matrix (Figs. 7 and 8). It was noted also that the Golgi lamellae on either side of the wound sometimes became more distinct than elsewhere. In the cells that survived the injury, the wound became smaller and the dark bands less phase-dark as time elapsed. In several cases, the wound diminished in size but remained ten minutes or more after the operation. In these instances, the wound seemed to be a hole through the whole thickness of the cell, the walls of which were supposed to consist of jellied Golgi matrix and/or newly formed cell membrane, since neither Golgi elements nor lipid droplets were observed to enter the phase-lucent area of the wound. That in case that the incision was not straight, but was in the form of a zigzag, the wound tended to remain unaltered for at least a few minutes, might be thought to be due to these circumstances.

When the Golgi lamellae which were vaguely visible in the Golgi area were pushed in one direction with a microneedle inserted between them, they were piled one upon another and returned to their original position when it was released. The operation could be repeated with the same result. Also, when the Golgi area was stretched with a microneedle thrusted in at its one pole and then. 
released, it resumed its original form, the Golgi lamellae in the area retaining their original position relative to one another ar relative to the lipid droplets lying on its outskirts. These observations are consistent with the view that the Golgi lamellae are embedded in a soft jelly-like medium. If a microneedle was gently and continuously moved in one direction, the lamellae appeared to turn aside from the course of the needle. There was no evidence either that they were cut into halves or that they were turned into fragments, which suggested that they were not brittle structures.

It was possible to move a lipid droplet in the Golgi area with the tip of a microneedle. It returned to the original position when the needle was withdrawn. It was also the case with a small fluid vacuole in the Golgi area. However, a large vacuole did not move when it was pushed, but changed from a spherical to an ovoid shape and resumed its original shape when released. Thus, there was good reason to presume that the Golgi area was semi-solid in consistency, but repeated disturbances with a microneedle were never followed by a thixotropical change.

Not a slight resistance was met with when the tip of a microneedle is moved through the boundaries of the Golgi area, from the inside of the area into the general cytoplasm or vice versa. The movement of the tip was not accompanied by any flow of the Golgi matrix or the general cytoplasm, nor by any inflow of one into the realm of the other. These observations are in agreement with the view that no firm structural boundary exists between the Golgi area and the general cytoplasm, and with the supposition that both of these are nearly equal in consistency, being a semi-solid gel. The following results of the experiments are also reconcilable with such a view.

It was possible to hold a lipid droplet of a moderate size with the tip of a microneedle and carry it through the Golgi area or/and through the general cytoplasm. This was often accompanied by cell death. But, the following experiment in a fibroblast was one of the cases in which the cell could remain alive during the whole course of the operation. A lipid droplet situated near the outer margin of the Golgi area was caught by a microneedle and was introduced into the Golgi area up to its distal end, piles of the Golgi lamellae in the way being displaced by the advancing droplet. These lamellae seemed, after having been altered only in form and position, to return after a while to their almost original condition (Figs. 9 and 10). The droplet was then detached from the tip of the needle. It 
went back a short distance, as if it were pushed, and stopped (Fig. 11). The same lipid droplet was again caught by the needle tip and carried out of the Golgi area (Fig. 12). The cell still remained alive, but was killed by a further treatment of pulling the lipid droplet out of the cell through the cell membrane. This experiment provided us with a further evidence for the fact that there is neither a great difference in consistency nor an actual barrier between the Golgi area and the general cytoplasm, and that the Golgi lamellae are not brittle structures. That the Golgi lamellae which had been displaced by the lipid droplet nearly resumed their original form and position might be attributed to the elasticity of the Golgi matrix rather than to their own.

(4) Other observations.

When a living cell was given a stimulus with the microneedle, the commonest reaction was a retraction and rounding up of the tip of pseudopodia, which appeared then like a phase-dark protuberance or a fleck (Figs. 8 and 14). Not only the periphery but the upper and lower surfaces of a flattened cell also possessed pseudopodia and showed many dark flecks (Figs. 10 and 12).

A less frequent reaction of the living cell to a rather severe injury was formation of phase-dark streaks in the cytoplasm which sometimes intertwined like a network. They were probably local thickenings or contractions of the cytoplasm. Phase-bright areas in the meshes of the phase-dark network of ten simulated fluid vacuoles.

When either the Golgi area or the general cytoplasm of a living cell is pricked with a microneedle, a few vacuoles were sometimes formed in the general cytoplasm in proximity to the site of injury. Also, vacuoles were formed in the Golgi area, when either this area or the neighboring general cytoplasm was injured (Figs. 13-16). Vacuoles once formed did not diminish in size nor disappeared, but continued to exist during the course of observation. Prior to the appearance of vacuoles, the point of attack by the microneedle often became phase-darker owing probably to a thickening of that part of the cytoplasm or the Golgi matrix or, in rarer cases, phase-darker streaks issued from that point to the neighboring cytoplasm.

Whether a cell is still alive or not can be judged by the reactions above described. One may be able to study, using these reactions as criteria, to what extent a cell can endure injuries.

A small tear in the cell membrane was healed as soon as it was produced by pricking with the needle tip. Also, when the cell membrane was successively broken at contiguous places, it did not 
necessarily cause cell death. However, an attempt to expel a lipid droplet, which had been caught by a microneedle, out of the cel: through the cell membrane unexceptionally brought death to the cell. On the other hand, an experiment to cut off a considerable portion of the cytosome, i.e., one fifth of the total length of the cell, caused a great shock reaction on the part of the cell, which, however, subsided and left the cell alive in an apparently healthy condition (Figs. 1 and 2).

An impression was obtained through the course of our experiments that the cytoplasm of a living cultured cell is a soft jelly in consistency. A vacuole of a medium or large size was altered in form when pushed by a blunt needle, but was usually again rounded when it was freed. A lipid droplet removed from its original position again returned to it when released. But, it could be, as above illustrated, displaced a considerable distance through the cytoplasm by a continued effort with the tip of a microneedle. A comparable attempt never succeeded after the death of a cell.

When a severe injury was given to the cytoplasm or immediately to the nucleus, granules or filaments appeared within the nucleus, the latter representing undoubtedly chromonema. It was also observed that about 10 minutes after the cytoplasm was severely injured, the nucleolus was disintegrated into fine filaments to be dispersed throughout the nucleus. Both of these alterations in the nucleus occurred in the cells not yet indicating any degenerative changes in the cytoplasm.

Structures identifiable as the cisternae of the endoplasmic reticulum were not usually visible in our materials. But in some cells, granular or reticular formations distinguishable from mitochondria were observed in the cytoplasm. They were lost from view when an injury was given to the cytoplasm to become again visualized after a short while. Conversely, in some other cells, in which the endoplasmic reticulum was hardly visible in the living condition, a cell injury and consequent cell death caused it to be readily recognizable in the cytoplasm.

\section{Discussion}

In the living cultured cells used in our present study, the Golgi complex appeared to consist of a cluster of phase-dark filaments or threads with blurred contour embedded in a matrix and was always situated adjacent to the nucleus. The filamentous structures proved 
in our previous study to be readily blackened with osmium tetroxide and evidently they represented elements of the classical Golgi apparatus. The membranes composing the lamellae of the Golgi complex are known to be usually thicker than the membranes of the endoplasmic reticulum. That the latter membranes in living cultured cells were visible in favorable conditions with the phase microscope was evidenced by $\mathrm{R}$ os e and $\mathrm{P}$ o m e r a t (1960), and the reason why such membranes could be seen with phase optics was explained by F a w cett and I to (1958) and I to (1962). The same reason may be applicable to the Golgi membranes or lamellae.

Regarding phase microscopic observation on isolated cells, Ba re r (1955) said, "In some cases in which the presence of a nuclear membrane can at first hardly be discerned, quite a sharp dark line may appear in the course of time. It is not altogether certain therefore whether all cells which appear to have a definite nuclear membrane are not in fact already slightly damaged." Fawcett and Ito (1958) also stated that in guinea pig testicular cells, dark contours of the cisternae of the endoplasmic reticulum increased in contrast, in number and in linear extent with increasing time after isolation of cells from the testis. Such an increase in contrast of the intracellular membranous structures was realized almost instantaneously in our experiments, when the cultured cells were severely injured with a microneedle, and may safely be attributed to irreversible changes in cellular organization leading to death. A rapid movement of the microneedle calling into action a strong tensile force in a greater part of the cytoplasm and a puncture of the nuclear membrane necessarily induced such irreversible changes that resulted in an increase in dark contours of the nuclear membrane, nucleolus, Golgi lamellae, mitochondria and other cytoplasmic inclusions including possibly the endoplasmic reticulum.

The morphology of the Golgi complex, which was visualized by causing cell death artificially, may be said to be most true to nature, since it was not accompanied by distortion or shrinkage inevitable in treatment with fixatives. In reality, it was composed of nothing other than the Golgi lamellae, which were only made distinctly visible by occasioning cell death with a mechanical means. They made their appearance within the premises of the Golgi area and in conformity with its variable shape. Most of the Golgi lamellae were arranged parallel to the nuclear membrane, but the rest evidently ran in various directions. The lamellae never appeared smooth but fluffy in outline. Further, careful observation with 
adjusting the focus of the microscope showed that they anastomosed with each other at many points, suggesting that they were not simply parallel stacks of flattened sacs as might be supposed from most of the published electron micrographs, but were flattened sacs communicating with each other as La cy (1957) described them in the neurones of Patella vulgata.

Distinction between the living and the dead cells was clearly evident under the phase microscope, the vague contours of the nucleus, nucleolus and cytoplasmic inclusions in the former, as above described, turning very distinct in the latter. One is thus able to perform micro-operations with ample confidence that the cells which are treated now are living ones. Alterations in the dead cells seem to have been observed only with difficulty by the early micrurgists working with the dark-field illumination: merely " a faintly perceptible increase in the opacity of the cells" was noted (C ha mbers and Fell 1931). The occurrence of dark flecks due to contraction of the pseudopodia and the fibrillation in the cytoplasm, which occurs more rarely, may also be used as a criterion that the cell under manipulation is living.

Bearing the above criteria of the living cells in mind, it was evident that a cut to a small extent either in the peripheral or the internal cytoplasm, a push against the nuclear membrane, a displacement of the nucleolus, or a displacement of cytoplasmic inclusions could be made without causing death of the cell in so far as they were done gently. A drastic tear accompanied with a stretch of the cytoplasm and a puncture of the nucleus unexceptionally caused cell death, as Chambers and Fell (1931) had indicated. We were unable to displace individual mitochondria with the tip of a microneedle. On the other hand, a lipid droplet could easily be displaced a considerable distance without a damage to the cell, but on being released from the needle tip, it went back a little to where it came from, providing an unequivocal evidence that the cytoplasm was a soft jelly that allowed a small object to move in it, but was elastic at the same time. In our experiment, in which a lipid droplet was once introduced into the Golgi area and then brought out of it in a living cell, it was made evident that the Golgi lamellae were elastic and not fragile, since they were once removed and then returned to their original position and apparently retained their original morphology.

The proposition that the Golgi double membranes or sacs are embedded in a specialized matrix ( $\mathrm{S} j$ öst $\mathrm{r}$ a nd and $\mathrm{H}$ a n z on 1954) 
has not come to be widely accepted by electron microscopists. Indeed, our results above described of various sorts of micro-operations suggested that the ground substance of the Golgi area is of a consistency like that of the general cytoplasm, no difference in reactions toward the attacks with a microneedle or the displacement of a lipid droplet being noted between the two. Mitochondria and lipid droplets are excluded from the Golgi area in most of the cells, because the Golgi area is occupied by the elements of the Golgi complex. But, lipid droplets are often, and mitochondria are in a few of the cells studied by vital staining with janus green, seen to be included there ( $\mathrm{T}$ a k a $\mathrm{g}$ i and $\mathrm{M}$ a s u d a 1956). In this connection, we are reminded of $\mathrm{H}$ i r s c h 's contention (1961) on electron microscopic evidence that the Golgi area, his "lamellar vacuolar field", is an open system, not sharply limited to the other basic substance of the cell.

It would be impossible under the present restrictions of micrurgical techniques to displace the Golgi complex as a whole in a living cell. In this respect, reference should be made to the famous experiment of $\mathrm{B}$ e a $\mathrm{m} \mathrm{s}$ and $\mathrm{K}$ i $\mathrm{n} \mathrm{g}$ (1934), in which the Golgi apparatus in the uterine gland cells moved in a manner characteristic of a viscid fluid toward the centripetal pole when centrifuged for 30 minutes at 400,000 times the force of gravity. It may be questioned whether the cells were still living during the course of the treatment. The report of Pollister (1939) that the Golgi apparatus, squeezed out from the Gerris spermatid into Ringer's solution, was unaltered for about an hour, deformed under pressure, but returned to the original shape when released, does not refer, of course, to the elasticity of the Golgi apparatus in the living cell. It seems amply evident from the results of our experiments that the Golgi complex consists of a semi-fluid matrix and semi-solid membranous elements, both of which are elastic.

Our observation that vacuoles are formed in some cases in the Golgi area in response to a stimulus is interesting with regard to $\mathrm{R}$ os e's observation (1961) of the appearance of secretion vacuoles in the Golgi complex in cultured osteoblasts. However, the formation of vacuoles in our cases seems to be of the same nature as that which occurs in the general cytoplasm as a reaction to excitation. It occurs in the living cell and differs from the formation of vacuoles in those cells which have commenced necrobiotic changes. The latter is due according to the recent investigation of $\mathrm{B} \mathrm{u} \mathrm{c} \mathrm{k} \mathrm{le} \mathrm{y}$ (1962) to a dilatation of the nuclear envelope and of cisternae of the endoplasmic reticulum. 


\section{Summary}

Micrurgical studies were made on the Golgi complex in the living cultured cells under the phase microscope. The Golgi complex, which is a cluster of filamentous or membranous elements with illdefined contour in the living cell, increases in contrast as the nuclear membrane, nucleolus, mitochondria and the other cytoplasmic inclusions accentuate visibility, when death changes are elicited by severely injuring the cell. The Golgi complex then appears like a cluster of phase-dark threads which entirely fill up the Golgi area. These threads are optical sections of membranous structures, most of which are arranged parallel to the nuclear membrane and are seemingly anastomosed with one another with processes.

Micro-operations could be made on cells which were assuredly living during the course of treatment in view of their appearance under phase contrast and of their reaction to stimuli. The operations with the tip of a microneedle of gently stirring the Golgi area, of providing a scar in the same area, and of introducing a lipid droplet in and out of, the Golgi area prove that the ground substance in which the Golgi complex is embedded is of the same physical state as the general cytoplasm, i. e., it is like a soft jelly in consistency, and that the Golgi lamellae are not fragile but flexible and elastic ones.

\section{References}

B a rer, R. 1955. Phase-contrast, interference-contrast, and polarizing microscopy. Ch. 3 in "Analytical Cytology", New York, Toronto, London.

$\mathrm{B}$ e a m s, H.W. and $\mathrm{King}$, R. L. 1934. The effect of ultracentrifuging upon the Golgi apparatus in the uterine gland cells. Anat. Rec. Vol. 59, pp. 363-371.

B u ckley, I.K. 1962. Cellular injury in vitro: phase contrast studies on injured cytoplasm. J. Cell Biol. Vol. 14, No. 3, pp. 401-420.

$\mathrm{Ch}$ a m bers, R. and Fe11, H. B. 1931. Micro-operations on cells in tissue cultures. Proc. Roy. Soc., London, s. B Vol. 109, pp. 380-403.

Ch èvremont, M. 1956. Cytologie et Histologie, Liège.

Dalton, A. J. and Felix, M.D. 1952. Lipochondria and the Golgi substance in epithelial cells of the epididymis. Nature Vol. 170, pp. 541-542.

Dalto n, A. J. and Felix, M.D. 1953. Studies on the Golgi substance of the epithelial cells of the epididymis and duodenum of the mouse. Am. J. Anat. Vol. 92, No. 2, pp. 277-305.

F a w cett, D.W. and I t o, S. 1958. Observations on the cytoplasmic membranes of testicular cells, examined by phase contrast and electron microscopy. J. Biophys. a. Biochem. Cytol. Vol. 4, No. 2, pp. 135-142.

Frederic, J. 1956. Contributions a l'étude du cytoplasme par la microscopie a fort grossissement avec le dispositif anoptral. (Photographies sur le vivant 
et aprés fixation osmique de cellules cultivées in vitro). Exptl. Cell Res. Vol. 11 , pp. $18-35$.

$\mathrm{Han}$ a oka, M. 1954. Phase contrast microscopic studies on the fine structure of the Golgi body in the plasma cell. Acta Haem. Jap. Vol. 17, No. 7, pp. 1-12.

Hirsch, G.C. 1961. Suggestions for a new look at the lamellar-vacuolar field or "Golgi complex" and their pattern of lower parts. Acta Med. Okayama Vol. 15, No. 5, pp. $289-293$.

It o, S. 1962. Light and electron microscopic study of membranous cytoplasmic organelles. In "Interpretation of Structure", Symposia of the Intern. Soc. for Cell Biology Vol. 1, New York and London, pp. 129-148.

L a c y, D. 1957. The Golgi apparatus in neurones and epithelial cells of the common limpet Patella vulgata. J. Biophys. a. Biochem. Cytol. Vol. 3, No. 5, pp. 779796.

Pollister, A.W. 1939. The structure of the Golgi apparatus in the tissues of Amphibia. Quart. J. Microsc. Sci. Vol. 81, pp. 235-271.

Rose, G.G. and Pomerat, C.M. 1960. Phase contrast observations of the endoplasmic reticulum in living tissue cultures. J. Biophys. a. Biochem. Cytol. Vol. 8, No. 2, pp. 423-430.

Pose, G.G. 1961. The Golgi complex in living osteoblasts. J. Biophysic. a. Biochem. Cytol. Vol. 9, No. 2, pp. 463-478.

$\mathrm{S}$ jöstrand, F.S. and $\mathrm{H}$ anz on, V. 1954. Electron microscopy of the Golgi apparatus of the exocrine pancreas cells. Experientia Vol. 10, pp. 367-368.

Takagi, S. and Masuda, H. 1956. Fine structure of the Golgi apparatus of the fibroblasts in vitro as revealed by phase microscopy and vital staining. Bull. Univ. of Osaka Pref., ser. B Vol. 6, pp. 33-41.

Takagi, S. 1958. Studies of the Golgi complex in the living cell. Jap. J. Exptl. Morph. No. 12, pp. 1-11.

Takagi, S., Kitada, J., Masuda, H. and Tagawa, M. 1961. A comparative study of staining and histochemical reactions of the components of Golgi complex of the fibroblasts in vitro and the ascites tumor cells. Cytologia Vol. 26, No. 3-4, pp. 448-459.

\section{Explanations of Figures}

All figures are phase-contrast photomicrographs of cultured cells, before and after they were micro-operated on in various ways.

Fig. 1. At the center, a cell cultured from chick liver. The Golgi area containing fine threads is situated on the upper side of the nucleus. $\times 750$.

Fig. 2. The same cell as in Fig. 1, one minute after part of its cytoplasm was cut off. The Golgi area shrinked following the contraction of the neighboring part of the cytoplasm. But the cell showed no other significant changes. It relaxed afterwards and was again normal in appearance.

Fig. 3. A cell cultured from chick liver. The Golgi area is situated on the lower right of the nucleus. $\times 650$.

Fig. 4. The same cell as in Fig. 3, fifteen minutes aftear the nucleus was punctured. The cell was killed and the cytoplasmic inclusions were now distinct. The Golgi area was shifted to the right of the nucleus and was now seen to contain bizarre threads or membranes, fluffy in outline and provided with anastomoses.

Fig. 5. At the center, a fibroblastic cell cultured from chick heart. The Golgi area, 
which was situated above the nucleus, had two arms embracing a cluster of lipid droplets. Death changes of the cell were induced by stirring these lipid droplets. $\times 850$.

Fig. 6. The same cell as in Fig. 5. The Golgi membranes made their appearance in conformity with the shape of the Golgi area shown in the previous figure.

Fig. 7. An epithelial cell cultured from chick liver. The Golgi area extends to the upper left of the nucleus. A longitudinal cut was made in the area. $\times 800$.

Fig. 8. The same cell as in Fig. 7. Local thickening of the cytoplasm occurred on either side of the cut. But, the cell showed no immediate changes other than retraction of processes, but it began to degenerate fifteen minutes after the operation.

Fig. 9. A fibroblastic cell cultured from chick heart. The Golgi area extends to the lower left of the nucleus and its lower boundary is roughly indicated by an array of lipid droplets. Two dense crowds of Golgi membranes are observable, one beneath the nucleus and the other beneath the large lipid droplet located on the lower left of the nucleus. $\times 850$.

Fig. 10. The same cell as in Fig. 9. The large lipid droplet was pulled by the tip of the needle till the lower boundary of the Golgi area was reached. The crowd of Golgi membranes originally beneath the droplet was dispersed, but was restored to its original situation. Note the retraction of cell processes and the formation of phase-dark protuberances.

Fig. 11. The same cell as in the previous figure, two minutes after the operation. The lipid droplet moved back a short distance. The cell was nearly recovered from the shock of the operation.

Fig. 12. The same cell as in the previous figure. The same lipid droplet was again caught by the micro-needle and pulled out of the Golgi area into the general cytoplasm. The boundary between the two offered no tangible resistance. The cell survived the second operation, but was killed by the third operation, in which the same lipid droplet was pulled out of the cell.

Fig. 13. A fibroblastic cell cultured from chick heart. It had a Golgi area to the right of the nucleus. $\times 750$.

Fig. 14. The same cell as in Fig. 13, soon after a small cut was made at the lower end of the Golgi area. A dark spot of condensation appeared near the site of the injury. Lobopodia or protuberances were formed on the cell surface.

Fig. 15. The same cell as in the previous figure, two minutes after the injury. While the protuberances on the cell surface began to disappear, the dark spot in the Golgi area increased in size.

Fig. 16. The same cell as in the previous figure, thirteen minutes after the injury. The dark spot moved to the middle of the Golgi area and was now seen to be accompanied with two small vacuoles. Later on, more vacuoles appeared in the Golgi area. 
Plate I
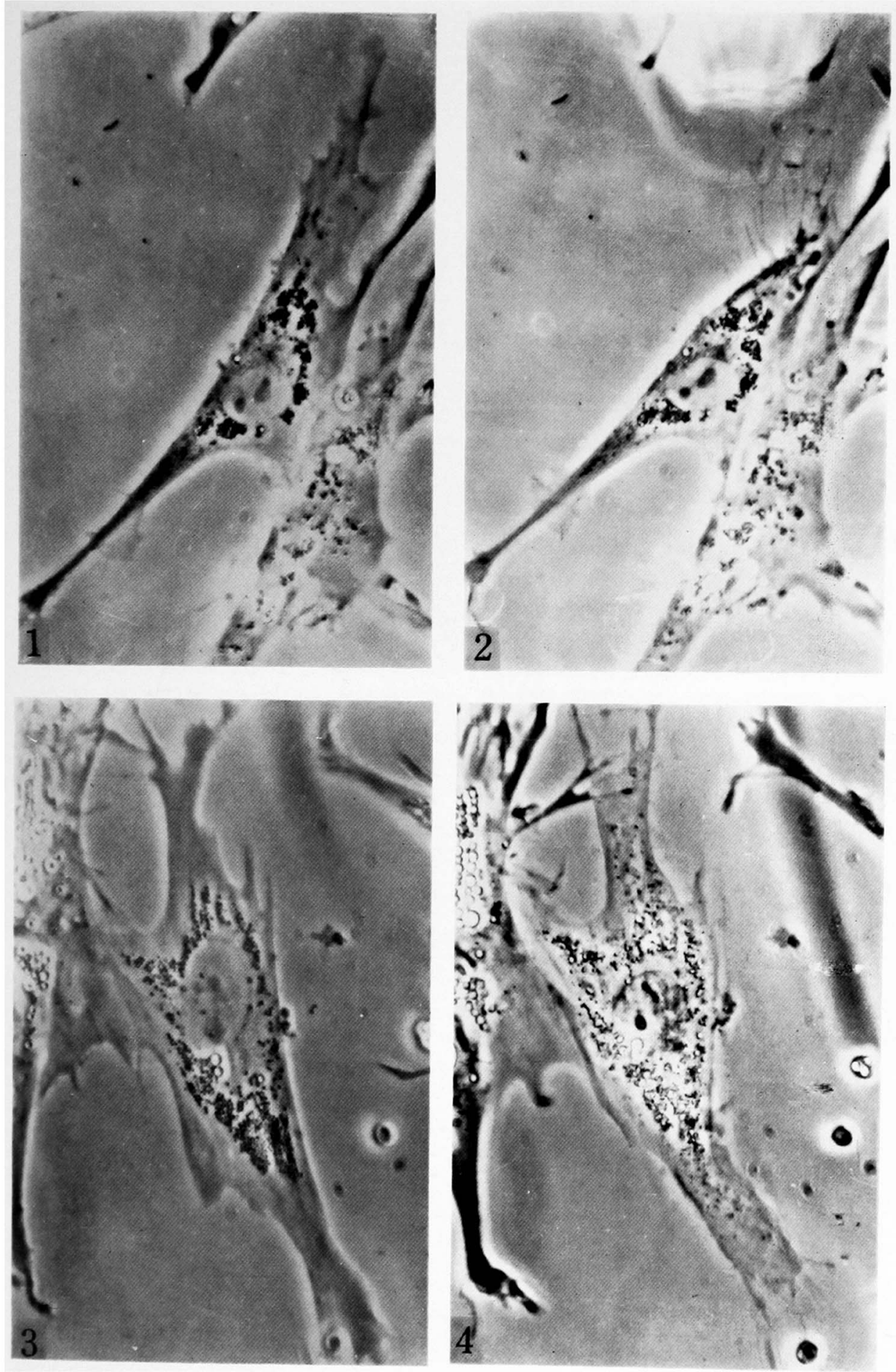

S. Takagi, H. Masuda and M. Tagawa 
Plate II
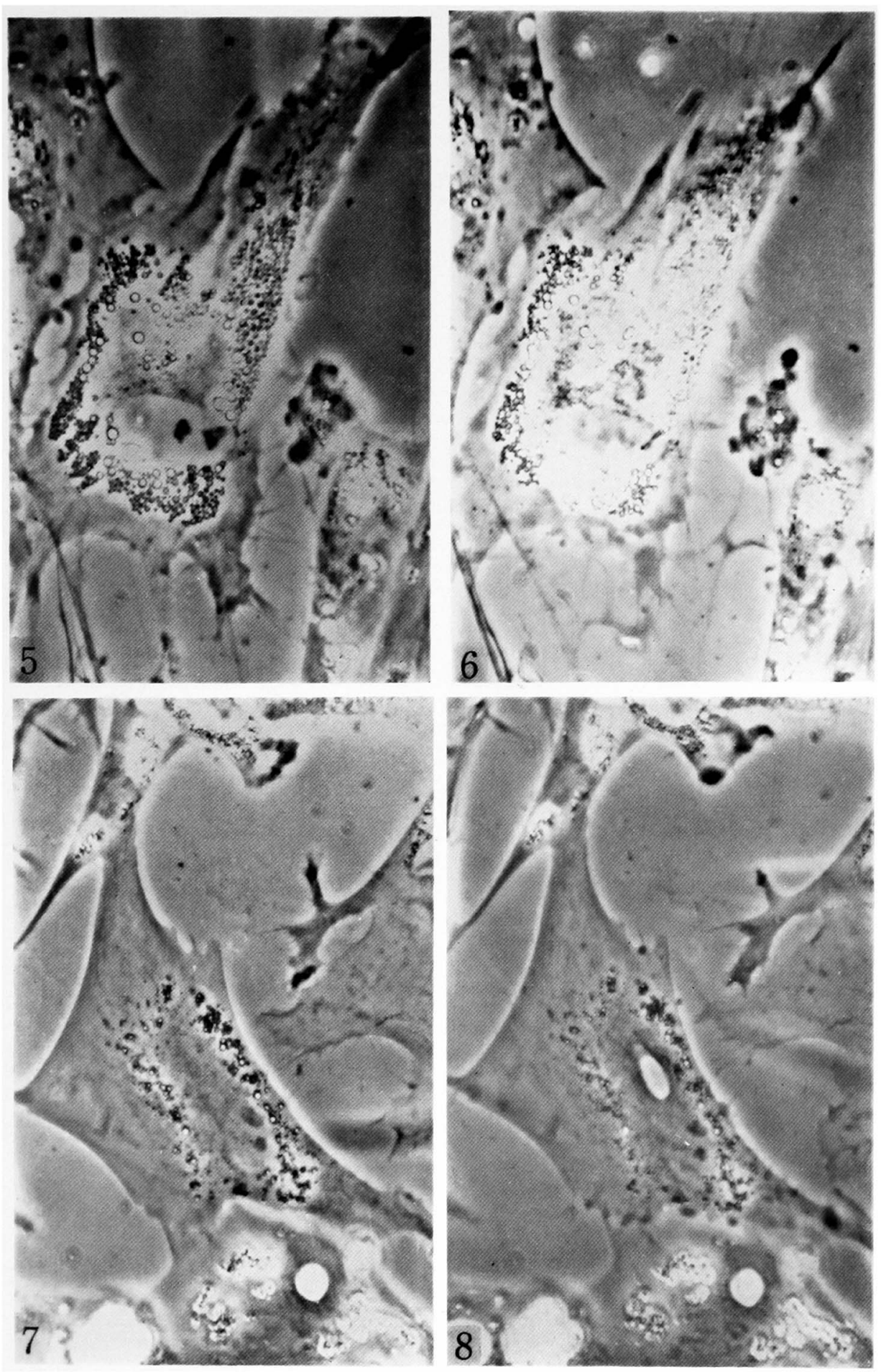

S. Takagi, H. Masuda and M. Tagawa 
Plate III
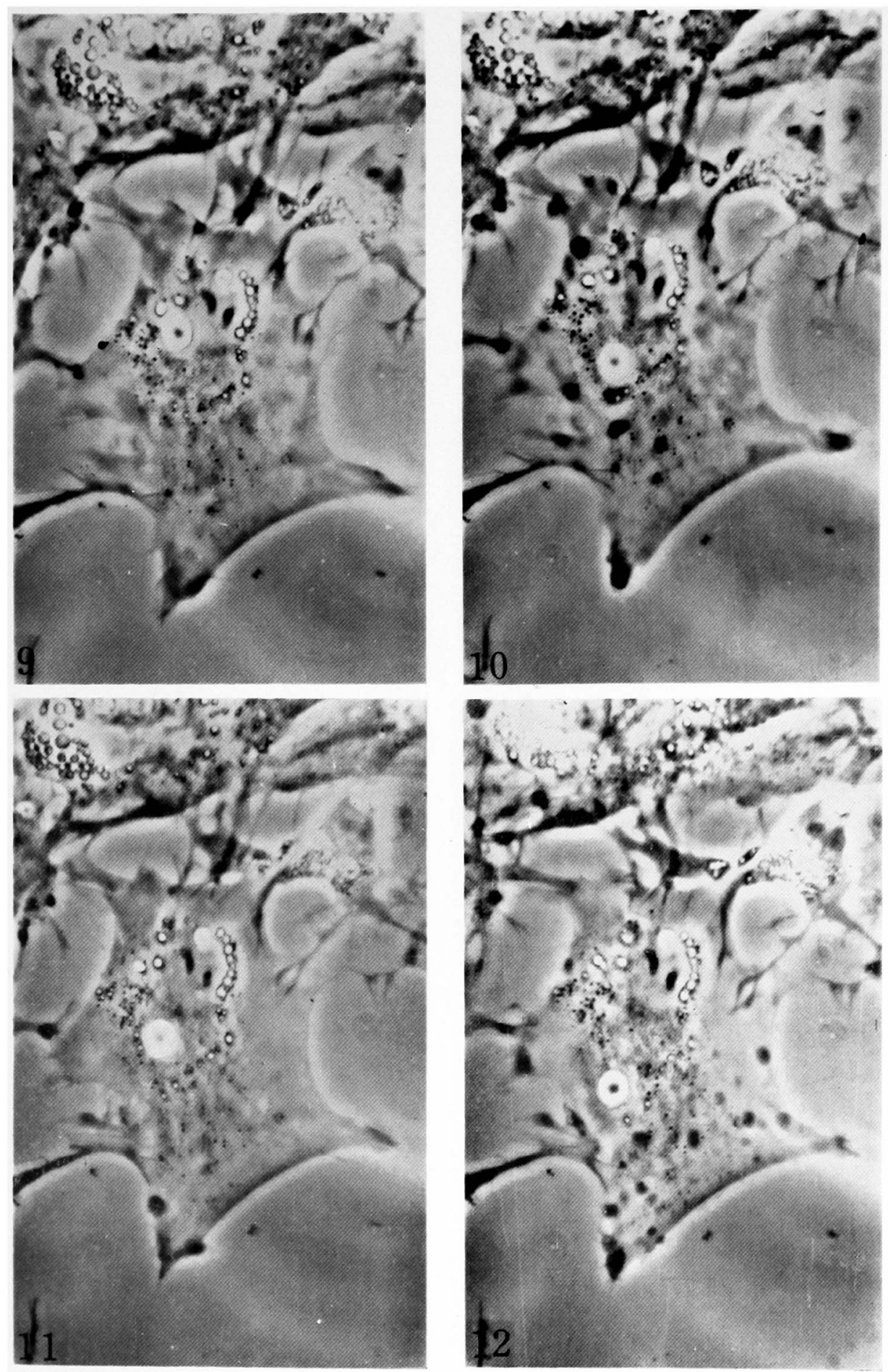

S. Takagi, H. Masuda and M. Tagawa 

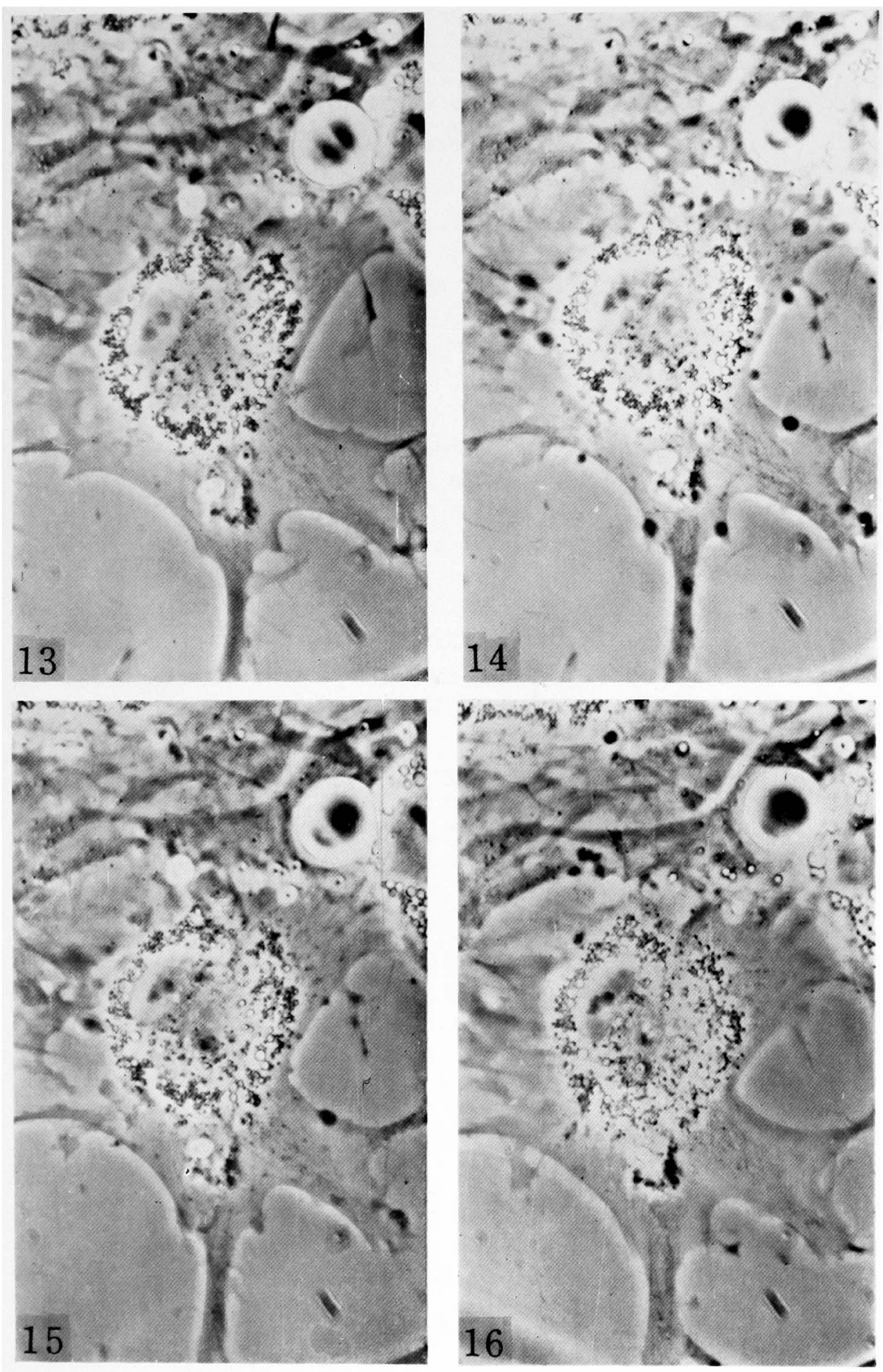

S. Takagi, H. Masuda and M. Tagawa 